\title{
Ovarian angiogenesis in polycystic ovary syndrome
}

\author{
Mariana Di Pietro, Natalia Pascuali, Fernanda Parborell and Dalhia Abramovich \\ Instituto de Biología y Medicina Experimental (IBYME-CONICET), Buenos Aires, Argentina \\ Correspondence should be addressed to D Abramovich; Email: dnabramovich@gmail.com
}

\begin{abstract}
Polycystic ovary syndrome (PCOS) is the most prevalent endocrine pathology among women in reproductive age. Its main symptoms are oligo or amenorrhea, hyperandrogenism and the presence of ovarian cysts. It is also associated with infertility, obesity and insulin resistance. Mainly due to its heterogeneity, PCOS treatments are directed to manage its symptoms and to prevent associated diseases. The correct formation and regression of blood vessels during each ovarian cycle is indispensable for proper follicular development, ovulation and corpus luteum formation. The importance of these processes opened a new and promising field: ovarian angiogenesis. Vascular alterations characterize numerous pathologies, either with increased, decreased or abnormal angiogenesis. In the last years, several anomalies of ovarian angiogenesis have been described in women with PCOS. Therefore, it has been suggested that these alterations may be associated with the decreased - or lack of - ovulation rates and for the formation of cysts in the PCOS ovaries. Restoration of a proper vessel formation in the ovaries may lead to improved follicular development and ovulation in these patients. In the present review, we attempt to summarize the alterations in ovarian angiogenesis that have been described in women with PCOS. We also discuss the therapeutic approaches aimed to correct these alterations and their beneficial effects on the treatment of infertility in PCOS.

Reproduction (2018) 155 R199-R209
\end{abstract}

\section{Introduction}

Polycystic ovary syndrome (PCOS) is the most prevalent endocrine pathology, affecting $5-10 \%$ of women in reproductive age (Norman et al. 2007). PCOS was first described in 1935 by Stein and Leventhal as a condition consisting of enlarged polycystic ovaries and amenorrhea (Stein \& Leventhal 1935). Since then, numerous studies have been performed to unravel the molecular mechanisms of PCOS and achieve better management of these patients. Besides, PCOS is a complex interplay between genetic and environmental factors (Rosenfield \& Ehrmann 2016). PCOS heterogeneity has opened an in-depth discussion that is still ongoing concerning its diagnosis and treatment. In 2003, a consensus was reached in Rotterdam to diagnose PCOS in women who present at least two of the following features: clinical or biochemical hyperandrogenemia, oligomenorrhea or amenorrhea and the presence of ovarian cysts in ultrasound (Rotterdam 2004). However, these features are usually accompanied by other comorbidities, such as increased risk of developing infertility, cardiovascular disease (CVD), metabolic syndrome, type-2 diabetes and endometrial cancer (Norman et al. 2007). Moreover, pregnant women with PCOS have increased risk of pregnancy complications, such as preeclampsia, miscarriage, gestational diabetes and preterm delivery (Norman et al. 2007). For all these reasons, PCOS is still a medical challenge. The current insufficiency of knowledge, eighty years after its first description, makes continuous research crucial to unravel the mechanisms involved in PCOS pathophysiology. Thus, there are still many controversies and discussion on PCOS treatments, which therefore target to manage its symptoms and to prevent associated diseases.

The female reproductive system presents periodic growth and regression of blood vessels together with changes in blood flow, with the ovary as one of the few adult tissues in which angiogenesis is an active process (Hazzard \& Stouffer 2000). Ovarian angiogenesis has proved to be an interesting field, since the correct formation and regression of vascular vessels during each cycle is indispensable for a proper follicular development, ovulation and corpus luteum formation. Small follicles are avascular and depend on stromal vessels for nutrition and hormone supply. When the thecal compartment begins to grow, blood vessels develop within this layer and each follicle relies on its thecal vascular network to survive and maturate (Hazzard \& Stouffer 2000). Formation and regression of the vasculature is tightly regulated by angiogenic factors synthesized mainly by ovarian cells. Vascular endothelial growth factor (VEGF) is the main angiogenic factor that promotes endothelial cell proliferation and migration and vascular permeability. It binds to VEGF receptor 2 (VEGFR2 or kinase insert domain receptor, KDR), which is expressed not only in endothelial cells, but also in granulosa and theca cells (Reynolds \& Redmer 1998, 
Abramovich et al. 2009). In addition, we have shown that VEGF acts directly on follicular cells independently of its angiogenic actions, regulating follicular cell function and fate (Irusta et al. 2010). VEGF can also bind to VEGFR1 (also known as fms-related tyrosine kinase 1, FLT1), which has a kinase activity one order of magnitude weaker than that of VEGFR2 (Waltenberger et al. 1994). Besides, numerous proteins promote endothelial cell proliferation and migration and act in coordination with VEGF, such as transforming growth factor B (TGFB1), basic fibroblast growth factor (bFGF) and placental growth factor (PIGF) (Pandya et al. 2006).

Transforming growth factor B (TGFB) belongs to the TGFB superfamily of proteins, which comprises activins, inhibins and the anti-Müllerian hormone $(\mathrm{AMH})$. There are three TGFB isoforms (TGFB1-3) that participate in the regulation of different cellular processes such as angiogenesis, proliferation and apoptosis in humans (Laiho et al. 1990, Yang \& Moses 1990, Satterwhite \& Moses 1994). Basic fibroblast growth factor (bFGF) is a potent angiogenic factor that promotes endothelial cell proliferation both in vivo and in vitro (Gospodarowicz et al. 1987). Due to its high affinity to heparan sulfate proteoglycans, it is sequestered to the extracellular matrix after secretion and needs to be released by proteases/heparinases in order to signal. Thus, bFGF acts mainly in a paracrine way near the secretion site (Turner \& Grose 2010). Placental growth factor (PIGF) is a member of the VEGF family that binds exclusively to the FLT1 receptor (Park et al. 1994). It is able to dimerize with VEGF to promote vessel growth (Cao et al. 1996).

The angiopoietin (ANGPT) system participates in the regulation of vascular stability and permeability by binding to the TIE2 receptor. ANGPT1 is the key member of this family regarding stability, since it promotes the maturation of newly formed blood vessels, contributing to quiescence and integrity of adult vasculature (Davis et al. 1996). ANGPT2 is a context-dependent agonist or antagonist of TIE2, which constrains vasculature stabilization (Maisonpierre et al. 1997, Shim et al. 2007). In the presence of VEGF, ANGPT2 triggers angiogenic sprouting by promoting detachment of mural cells, an essential step for endothelial cell proliferation, tubule formation and vessel growth. In the absence of VEGF, ANGPT2 promotes vascular destabilization and regression (Gale et al. 2002). Furthermore, we have observed that protein expression of ANGPT1, ANGPT2 and their receptor TIE2 increase during follicular development in the rat ovary (Abramovich et al. 2009).

Platelet-derived growth factors (PDGFs) belong to a protein family that encompasses five ligands (PDGFAA, PDGFBB, PDGFAB, PDGFCC and PDGFDD) and two tyrosine kinase receptors (PDGFRA and PDGFRB). The receptor involved in angiogenesis regulation is the PDGFRB, which only binds to the PDGFBB and PDGFDD ligands. Therefore, these two ligands are crucial factors involved in the regulation of vessel formation by inducing pericyte and smooth muscle cell recruitment to the newly formed vessels, promoting their maturation and stability (Hoch \& Soriano 2003, Betsholtz 2004). PDGFBB and PDGFDD are widely expressed in platelets, smooth muscle cells and endothelial cells (Heldin \& Westermark 1999), while PDGFRB is mainly expressed in fibroblasts, pericytes and vascular smooth muscle cells (VSMCs) (Nissen et al. 2007). In physiological conditions, protein expression of PDGF family members has been previously detected in the rat, mouse and human ovaries (Sleer \& Taylor 2007, Pinkas et al. 2008). In this regard, we have shown that PDGF system is necessary for follicular development induced by gonadotropins (Pascuali et al. 2015).

The entire angiogenic process is also regulated by antiangiogenic factors that inhibit endothelial cell proliferation and migration and control the end of the process, avoiding an excessive growth of blood vessels and the formation of tortuous vasculature. The main antiangiogenic factors are thrombospondins (TSP), a family that comprises five members (TSP 1-5), endostatin and angiostatin (Pandya et al. 2006). Soluble receptors, as soluble FLT1 (sFLT1) also act as antiangiogenic factors by trapping pro-angiogenic proteins and decreasing their bioavailability (Pandya et al. 2006).

In adults, most of the vasculature is quiescent and physiological angiogenesis occurs only in certain tissues, such as reproductive tissues and in wound healing. A myriad of pathologies display either increased angiogenesis (psoriasis, ocular neovascularization, rheumatoid arthritis and others), decreased angiogenesis (impaired wound or ulcer healing, myocardial ischemia, atherosclerosis, cerebral ischemia and others) or abnormal angiogenesis (telangiectasias, tumor vessels, preeclampsia, PCOS and others) (Ollauri-lbanez et al. 2017). Thus, targeting angiogenesis has become a growing therapeutic strategy in many of those disorders.

Recent studies have proven that there exist several angiogenesis anomalies in the ovaries of women with PCOS (Fig. 1). Moreover, it has been observed that these anomalies modify ovarian blood flow and possibly irrigation of follicles within the ovaries, leading to abnormal oxygen, nutrient and hormone supply. Hence, these vascular alterations may be partly responsible for deficient ovulation rates and for the formation of cysts in PCOS ovaries. Thus, it is not surprising that improvement in blood vessel formation has emerged as a new approach in PCOS fertility treatment.

\section{Ovarian vascularization in PCOS}

The first reports that assessed ovarian blood flow in PCOS are from Battaglia and coworkers (1995) and Zaidi and coworkers (1995). In those studies, the authors compared ovarian blood flow in control and PCOS patients by color Doppler analysis. Women were diagnosed with PCOS when they had oligo or 
Control

\section{Normal Angiogenesis}

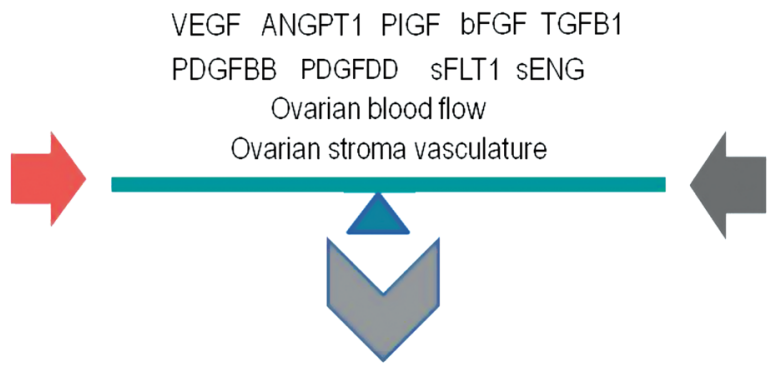

\section{Normal Folliculogenesis}

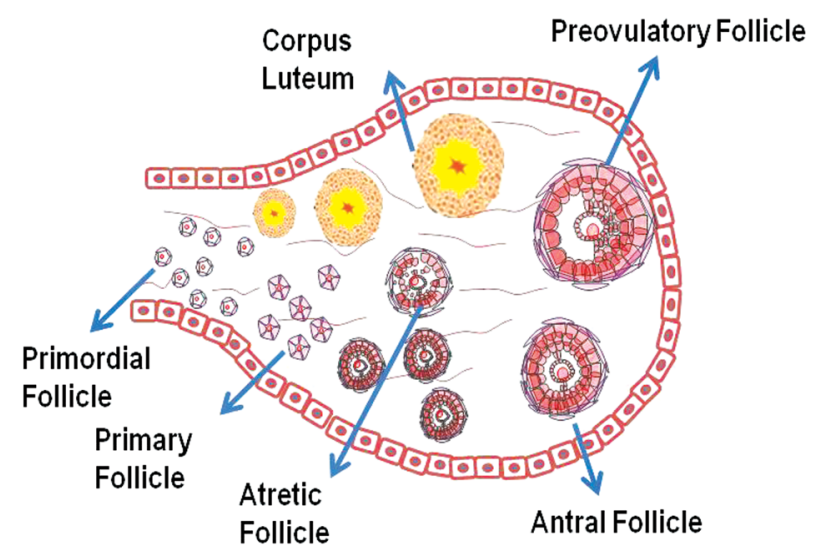

$\underline{\mathrm{PCOS}}$

\section{Altered Angiogenesis}

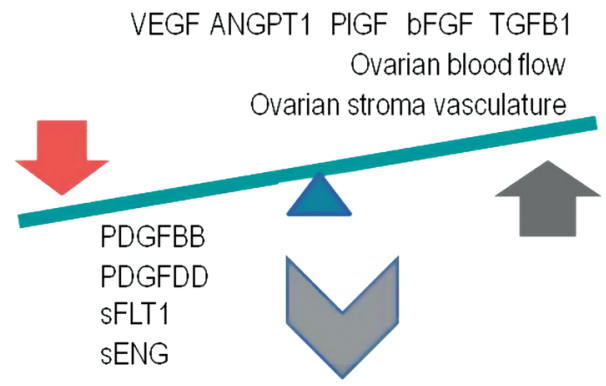

Altered Folliculogenesis

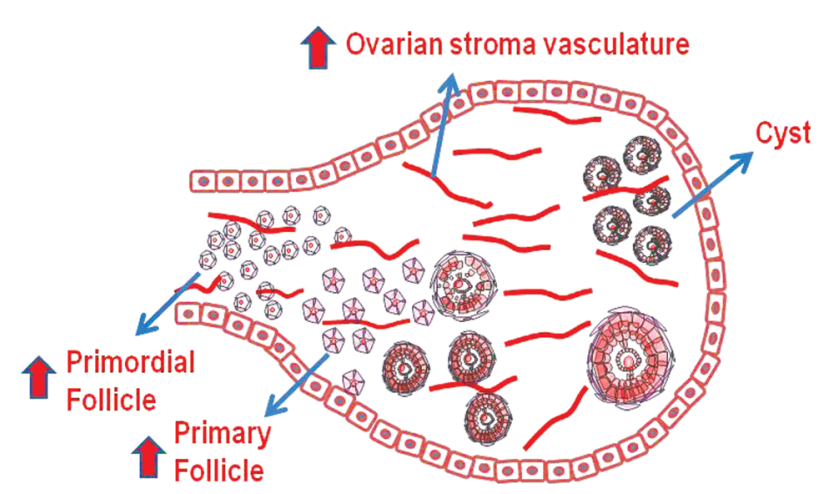

Figure 1 Deregulation of ovarian angiogenesis contributes to abnormal follicular development in women with PCOS. Ovarian angiogenesis is a tightly regulated process that needs a delicate balance of angiogenic factors, which is disrupted in women with PCOS, who present anomalous ovarian blood flow and angiogenesis. This altered angiogenesis may contribute to the ovarian features of PCOS such as abnormal follicular development, increase in the quantity of small follicles and failure in the selection of the dominant follicle, with anovulation and cyst formation. The figure summarizes the main angiogenic and ovarian alterations observed in women with PCOS.

amenorrhea, acne or seborrhea, hirsutism, increased $\mathrm{LH} / \mathrm{FSH}$ ratio and enlarged ovaries with a high number of subcapsular follicles. Interestingly, they found an increase in ovarian stromal vascularization, an increase in the pulsatility index $(\mathrm{Pl})$ and a decrease in the resistance index (RI) in the ovaries from patients with PCOS compared to control women. The authors hypothesized that ovarian neoangiogenesis may occur to a higher extent in these patients than in control patients (Battaglia et al. 1995, Zaidi et al. 1995). One of the studies also suggested that the increase in stromal vascularization may be involved in the increased risk of developing ovarian hyperstimulation syndrome (OHSS) and could serve as a diagnostic criterion for PCOS (Zaidi et al. 1995). One of those research groups then compared obese PCOS patients with lean PCOS patients and oligo vs amenorrheic PCOS patients. These authors have found that vascularization and Doppler parameter abnormalities increase with BMI and amenorrhea, in comparison with women with a milder PCOS phenotype
(Battaglia et al. 1996, 1997). Several reports assessed ovarian and uterine blood flow in PCOS patients and, despite their differences regarding the Doppler indices analyzed, all studies confirmed the increase in ovarian stroma vascularization regardless of the methodology used (Agrawal et al. 1998a,b, Resende et al. 2001, Abd El Aal et al. 2005, Carmina et al. 2005, Alcazar \& Kudla 2012). The study authored by Agrawal and coworkers drew especial attention to the relationship between the increase in stroma vascularization in PCOS with an increase in the levels of VEGF and the risk of developing OHSS in these patients (Agrawal et al. 1998a). That report was the first to recognize the influence of altered ovarian vascularization on systemic alterations seen in the PCOS patients. In the last years, another report evaluated these parameters by using a 4D spatiotemporal image correlation-high definition flow transvaginal ultrasound approach. In this study, PCOS was defined according to the Rotterdam criteria. Once again, the authors found evidence of higher vascularization and 
lower impedance to flow in ovarian stromal vessels from women with PCOS (Alcazar \& Kudla 2012).

There are considerable differences among these reports concerning the methodology used to assess ovarian blood flow and also the PCOS diagnosis criteria, which has suffered many changes through the years and consensus meetings. Nonetheless, they have all demonstrated the same alterations, which strengthen the relevance of the angiogenic component in women with PCOS.

\section{Angiogenic factors in PCOS}

\section{Vascular endothelial growth factor (VEGF)}

Modifications in the VEGF family were the first and best-described angiogenic alterations in the ovaries from women with PCOS. Early in 1995, Kamat and coworkers found an increase in VEGF expression in granulosa, theca and luteal cells in ovarian tissue from PCOS patients analyzed by immunohistochemistry (Kamat et al. 1995). Originally, the aim of their study was to analyze the presence of VEGF in other ovarian compartments besides the corpora lutea. However, as they included three patients with PCOS and patients that had indication for surgery for other pathologies as a control group, they were able to describe that PCOS patients had an increased protein expression of VEGF. Afterwards, that preliminary result was confirmed by various groups. Agrawal and coworkers found a positive correlation between the levels of serum VEGF and ovarian blood flow, providing the first explanation for the increase in stromal vascularization in PCOS ovaries (Agrawal et al. 1998b). Increased levels of VEGF were associated with the increase in ovarian blood flow, vascularization and OHSS by many authors (Ferrara et al. 2003, Abd El Aal et al. 2005, Gomez et al. 2010, Scotti et al. 2014). Interestingly, we and other authors have demonstrated that the protein concentration of VEGF in follicular fluids (FF) was higher than the serum concentration, suggesting that the source of increased serum VEGF is the ovary (Artini et al. 2006, 2009, Scotti et al. 2014).

Soluble Fms-like tyrosine kinase 1 (sFLT1) is a soluble receptor that binds to VEGF and blocks its interaction with the membrane receptor, regulating VEGF bioavailability. Patients with PCOS present lower serum and FF sFLT1 concentration than control patients, making VEGF bioavailability in PCOS patients even higher (Artini et al. 2009, Tal et al. 2014).

Different VEGF gene polymorphisms have been described in the promoter, intronic and untranslated regions, some of them associated with differences in the levels of VEGF protein expression (Vural et al. 2009). Therefore, several studies have evaluated VEGF polymorphisms in women with PCOS in different populations. In the Korean population, single-nucleotide polymorphisms (SNP) at +9812 and +13553 sites are likely to be associated with PCOS (Lee et al. 2008). In South Indian women, the VEGF $+405 \mathrm{G} / \mathrm{C}$ polymorphism is associated with an increased risk of PCOS (Guruvaiah et al. 2014). Almawi and coworkers showed a significant relationship between the $-583 \mathrm{~T}>\mathrm{C}$ and the $-634 \mathrm{G} / \mathrm{C}$ variants and PCOS (Almawi et al. 2016) in Bahraini population. Although these results are interesting and promising, further investigations with larger study groups and with unrelated populations are needed to fully associate those polymorphisms to the PCOS phenotype.

Most of these alterations have also been observed by us and other authors in animal models of PCOS, such as postnatal DHEA injection or postnatal estradiol valerate injection in rats (Abramovich et al. 2012, Karimzadeh et al. 2013, Di Pietro et al. 2015, 2016). Despite their differences with the human pathology, these animal models are useful preclinical ways to study ovarian angiogenesis in PCOS and the potential interventions to manage it. So far, angiogenic factor levels in other PCOS animal models have not been assessed. In a DHEA rat model of PCOS, we showed increased ovarian VEGF protein levels and decreased FLK1 protein levels (Abramovich et al. 2012). Expression levels of the main VEGF membrane receptor in the ovaries of women with PCOS have not been studied yet.

Taken together, all these studies highlight the relevant role of ovarian VEGF alterations in the pathogenesis of PCOS. Moreover, VEGF emerges as a possible therapeutic target for follicular development and ovulation improvement in these patients.

\section{Basic fibroblast growth factor (bFGF)}

As previously stated, bFGF promotes endothelial cell proliferation, migration and survival (Turner \& Grose 2010). Regarding its relationship with PCOS, only one work analyzed the levels of bFGF in serum and FF from women with PCOS (Artini et al. 2006). The authors reported increased serum bFGF protein in PCOS patients undergoing ovarian stimulation with gonadotropins compared to control patients, only at day of hCG administration, as well as increased bFGF in FF. It is interesting that the authors have not found differences in bFGF levels between PCOS and control patients before $\mathrm{FSH}$ stimulation. The increment in serum bFGF in PCOS patients was found only after FSH stimulation, suggesting that FSH may be inducing bFGF expression differentially among PCOS and control women (Artini et al. 2006). More studies are needed to further explain these findings.

These findings indicate that bFGF deregulation plays an important role in PCOS ovaries after FSH stimulation. Increased bFGF levels could be involved in the higher risk of patients with PCOS to develop OHSS. 


\section{Transforming growth factor $B$ (TGFB)}

An increasing number of studies have addressed TGFB deregulation in patients with PCOS, since they regulate diverse biological processes such as angiogenesis, cell proliferation and tissue fibrosis (Laiho et al. 1990, Yang \& Moses 1990, Satterwhite \& Moses 1994), all of them related to PCOS. It has been established that TGFB1 is increased in serum from patients with PCOS compared to control patients (Raja-Khan et al. 2010, Tal et al. 2013b, Liu et al. 2015). Moreover, soluble endoglin (sENG), a soluble receptor that binds to TGFB1 and 3, is decreased in serum of patients with PCOS, leading to higher TGFB1 bioavailability (Tal et al. 2013b).

These results suggest that increased serum TGFB1 levels and bioavailability may contribute to augmented ovarian angiogenesis and a greater risk of ovarian hyperstimulation after gonadotropin administration in these patients.

\section{Placental growth factor (PIGF)}

Tal and coworkers (2014) analyzed PIGF protein levels in serum and FF from women with PCOS and concluded that, while no differences were found in serum levels between control and PCOS patients, this factor is increased in FF from patients with PCOS. Moreover, due to a decrease in sFLT1 in FF, PIGF bioavailability was also increased in FF (Tal et al. 2014). These findings suggest a pertinent role for PIGF in ovarian angiogenesis deregulation in PCOS. However, more studies should be conducted to confirm the involvement of PIGF in this pathology.

\section{Angiopoietins (ANGPTs)}

The levels of ANGPT1 and 2 have been found altered in patients with PCOS, as well as in animal models of PCOS. Sova and coworkers evaluated serum levels of ANGPT2 protein in control and PCOS patients and found no differences between these two groups (Sova et al. 2010). This result was confirmed by Tal and coworkers when assessing the protein levels of ANGPT1 and 2 in both serum and FF from control and PCOS patients (Tal et al. 2013a). Nevertheless, they did report an increase in ANGPT1 with no changes in ANGPT2 in serum from patients with PCOS. In FF from patients with PCOS, ANGPT2 was increased with no changes in ANGPT1, leading to a higher ANGPT2/ ANGPT1 ratio, which correlates with an increase in vascularization. In our laboratory, we investigated FF ANGPTs levels and observed an increase in ANGPT1 with no changes either in ANGPT2 or in soluble TIE2 (Scotti et al. 2014). In those patients, VEGF was also higher in FF compared to control women. We proposed that elevated ANGPT1 may be compensating for the increase in permeabilization elicited by the high VEGF concentration (Scotti et al. 2014). The differences among these studies may respond to variations in stimulation protocols or to diversity in the population included in the study. Further studies are needed to determine whether the dynamic pattern of expression of ANGPTs in the ovary is altered in PCOS patients.

A recently published study examined the differences between women with PCOS that ovulated after clomiphene citrate (CC) and PCOS women resistant to CC (Wang et al. 2017). By using a cytokine antibody arrange confirmed by ELISA, one of the differences found was the circulating ANGPT2 levels, since PCOS women resistant to CC presented lower levels of ANGPT2 than sensitive ones. The authors conclude that CC-resistant women had an excessive ovarian angiogenesis that compromise their responsiveness to ovulation induction (Wang et al. 2017).

Furthermore, in a PCOS rat model developed by DHEA injection, we found an increase in ANGPT1, a decrease in ANGPT2 and an increase in ovarian TIE2 membrane receptor (Abramovich et al. 2012). The levels of ovarian TIE2 have not been studied in PCOS women yet.

Taken together, all these studies demonstrate that the ANGPT system is altered in PCOS, which may be related to the deregulation of ovarian angiogenesis in this pathology.

\section{Platelet-derived growth factor (PDGF)}

Among the PDGF family, PDGFRB, which binds to the PDGFBB and PDGFDD ligands, is the main receptor involved in angiogenesis, as described in the previous section. For this reason, we studied the levels of PDGFBB and PDGFDD in FF of PCOS women that undergo assisted reproductive techniques (ART) and found that both proteins were decreased compared to control women (Scotti et al. 2014). Moreover, in a DHEA rat model of PCOS, we also observed decreased levels of ovarian PDGFBB, PDGFDD and PDGFRB (Di Pietro et al. 2015). It is important to mention that PDGFRB signaling has been linked to the regulation of early folliculogenesis (Nilsson et al. 2006, Sleer \& Taylor 2007, Pinkas et al. 2008), since granulosa cells from primordial follicles express this receptor and PDGF stimulation promotes primordial to primary follicle transition (Nilsson et al. 2006). Hence, decreased levels of ovarian PDGF proteins in PCOS may not only be associated to deregulation of ovarian angiogenesis but also to the abnormal small follicle accumulation observed in these patients (Scotti et al. 2014).

Because of its involvement in primordial follicle activation and in newly formed vessel maturation, PDGF deregulation in PCOS is worth being investigated since its normalization may be directly connected to these two key processes, which are altered in this pathology. 
Therefore, PDGF is a good candidate to target in a future therapy for fertility improvement.

\section{Antiangiogenic factors in PCOS}

As angiogenesis is a complex process extremely regulated by factors that promote vessel growth and factors that inhibit this growth, it is not surprising that deregulation of blood vessel formation and proangiogenic factor levels is accompanied by alterations in antiangiogenic factors in PCOS. In this regard, some factors involved in physiologic inhibition of new vessel formation have been analyzed in patients and in animal models of PCOS.

As previously mentioned, sFLT1 is a soluble form of the VEGF receptor FLT1. It carries only six immunoglobulin domains of the full length form by alternative splicing (Kendall et al. 1996) and binds with high affinity to VEGF and PIGF, blocking their signaling through other receptors (Kendall et al. 1996). The levels of this soluble receptor have been found decreased in serum and FF of patients with PCOS (Artini et al. 2009, Tal et al. 2014), exacerbating the increase in its ligand levels.

Ithas been demonstrated that thrombospondin 1 (TSP1), a heparin-binding protein with potent antiangiogenic activity, is decreased in serum and subcutaneous and omental adipose tissue from patients with PCOS (Tan et al. 2009). This decrease is independent of either body mass index or PCOS phenotype, suggesting a role for TSP1 in the angiogenic alterations in this syndrome (Liu et al. 2015).

No other antiangiogenic molecules have been studied in PCOS, opening a big field in PCOS research since these compounds could serve as therapeutic tools to improve ovarian angiogenesis. Moreover, evidence of deregulation of antiangiogenic factors in combination with deregulation of proangiogenic factors strengthens the importance of the angiogenic process in the pathophysiology of PCOS. Table 1 summarizes the alterations in pro and antiangiogenic factors reported in PCOS.

\section{Therapeutic strategies to restore a correct ovarian angiogenesis}

The concept of restoring altered angiogenesis as a potential therapy has seen increasingly rapid advances in many pathologies, such as cancer (Albiges et al. 2011, Leite et al. 2011, Sharma et al. 2011, Socinski 2011), inflammatory diseases, retinopathies and agerelated macular degeneration (Bauditz \& Lochs 2007, Mitchell 2011, Ribeiro et al. 2011). As abnormal ovarian angiogenesis is a main feature of the PCOS, restoring this process has been proposed as a possible PCOS therapy to improve ovulation and fertility. To this end, many studies have analyzed the effect of different therapeutic approaches on ovarian angiogenesis (Table 2).

Laparoscopic ovarian drilling (LOD) is indicated in women with anovulatory PCOS that are resistant to clomiphene citrate (Thessaloniki 2008, Hueb et al. 2015). It consists of multiple ovarian punctures performed by laser or diathermy (2008). This treatment decreases $\mathrm{LH}$ and androgen levels and increases $\mathrm{FSH}$ in serum (Greenblatt \& Casper 1987). Although the exact mechanisms remain unknown, LOD improves ovulation in these patients (Hueb et al. 2015). Regarding angiogenesis, LOD reduces serum VEGF (Amin et al. 2003, El Behery et al. 2011) and ovarian blood flow velocities (Amin et al. 2003, El Behery et al. 2011, Elmashad 2011, Giampaolino et al. 2017) in women with PCOS, leading to a decrease in the risk of developing OHSS if these patients need to be stimulated with gonadotropins after LOD (El Behery et al. 2011). Therefore, restoration of normal ovarian VEGF levels and blood flow could be one of the mechanisms that contribute to ovulation in these patients.

Another approach that aims to improve ovarian angiogenesis in PCOS is the use of the biguanide metformin (MET). MET is an oral hypoglycemic drug, widely spread for the treatment of type 2 diabetes. Despite the controversy of using MET to improve fertility and/or ART success in women with PCOS, MET has been reported to enhance fertility parameters, including live

Table 1 Alterations in angiogenic factors in women with PCOS.

\begin{tabular}{llll}
\hline Factor & $\begin{array}{l}\text { Increased/ } \\
\text { decreased }\end{array}$ & Measurement & Studies \\
\hline VEGF & Increased & Serum, follicular fluid, ovary & Kamat et al. (1995), Agrawal et al. (1998a,b), Artini et al. (2006, 2009), Scotti et al. (2014) \\
PIGF & Increased & Follicular fluid & Tal et al. (2014) \\
bFGF & Increased & Serum and follicular fluid post hCG & Artini et al. (2006) \\
TGFB1 & Increased & Serum & Raja Khan et al. (2010), Tal et al. (2013b), Liu et al. (2015) \\
ANGPT1 & Increased & Serum & Tal et al. (2013a) \\
PDGFBB & Decreased & Follicular fluid & Scotti et al. (2014) \\
PDGFDD & Decreased & Follicular fluid & Scotti et al. (2014) \\
sFLT1 & Decreased & Serum, follicular fluid & Artini et al. (2006), Tal et al. (2014) \\
TSP1 & Decreased & Serum, adipose tissue & Tan et al. (2009, 2010) \\
sENG & Decreased & Serum & Tal et al. (2013b) \\
\hline
\end{tabular}

ANGPT1, angiopoietin 1; bFGF, basic fibroblast growth factor; hCG, human chorionic gonadotropin; PDGFBB, platelet-derived growth factor BB; PDGFDD, platelet-derived growth factor DD; PIGF, placental growth factor; sFLT1, soluble FLT1 receptor; sENG, soluble endoglin; TGFB1, transforming Growth Factor; TSP1, Thrombospondin1; VEGF, Vascular Endothelial Growth Factor. 


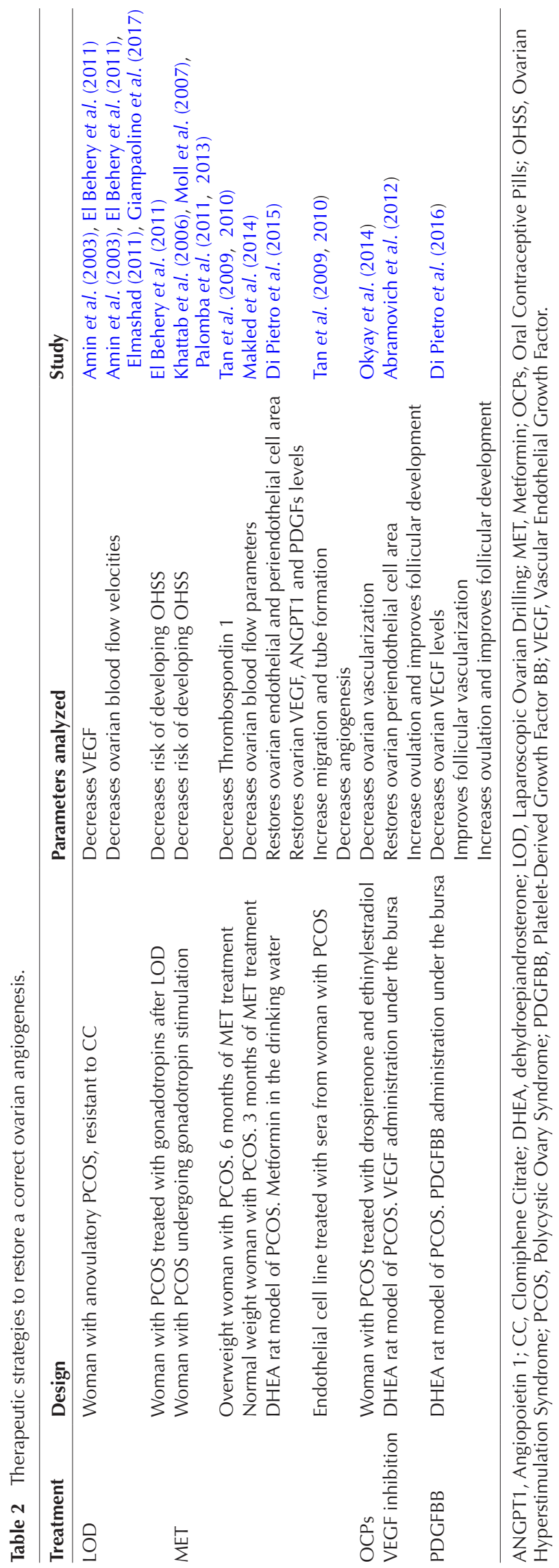

birth rate in some PCOS populations (Jungheim \& Odibo 2010, Johnson 2011, Kjotrod et al. 2011, Tang et al. 2012, Palomba et al. 2013, 2014). Moreover, MET can regulate angiogenesis in different in vivo and in vitro models. For example, in ovarian cancer, MET was proposed as an antiangiogenic adjuvant to other therapies due to its capability of decreasing VEGF (Rattan et al. 2011, Liao et al. 2012, Shank et al. 2012, Wu et al. 2012). MET was also able to decrease angiogenesis in murine models of diabetes and obesity, thus preventing vascular damage (Abdelsaid et al. 2014, Dallaglio et al. 2014). Moreover, MET prevented OHSS in PCOS and non-PCOS women undergoing gonadotropin stimulation for ART (Khattab et al. 2006, Moll et al. 2007, Palomba et al. 2011, 2013). For these reasons, many researchers have started to evaluate the involvement of MET on regulation of ovarian angiogenesis in women with PCOS.

Tan and coworkers studied the effect of MET in an overweight PCOS population compared to overweight women without PCOS (Tan et al. 2009, 2010). These authors found that MET restores the decreased levels of serum and adipose tissue TSP1 in women with PCOS after 6 months of treatment. Additionally, while sera from women with PCOS increase migration and tube formation in an endothelial cell line, MET decreases these effects on in vitro angiogenesis (Tan et al. 2009, 2010). In normal weight women with PCOS, Makled et al. have shown that 3 months of MET treatment reduces ovarian blood flow parameters together with ovarian volume and corrects hormonal profiles (Makled et al. 2014). Whether the improvement in hormonal levels is a cause or a consequence of the improvement in ovarian stromal vascular bed still needs to be addressed.

In order to determine the mechanisms involved in MET regulation of ovarian angiogenesis, we evaluated the effect of MET in ovarian angiogenesis in a rat model of PCOS developed by prepubertal injection of DHEA (Di Pietro et al. 2015). We found that MET restores the ovarian endothelial and periendothelial cell area and the ovarian VEGF, ANGPT1 and PDGFs protein levels. MET also improves follicular development in the PCOS animals, decreasing ovarian cysts and increasing ovulation (Di Pietro et al. 2015). Since MET treatment has led to promising results regarding angiogenesis restoration, further studies are worth pursuing to deepen these findings.

Oral contraceptive pills (OCPs) are the first line of treatment after lifestyle modifications for women with PCOS who do not seek pregnancy (Vrbikova \& Cibula 2005). OCPs decrease $\mathrm{LH}$ and androgen synthesis and increase sex hormone binding globulin (SHBG), leading to a decrease in the free androgen index (FAI) (Nader \& Diamanti-Kandarakis 2007). Okyay et al. have observed that a 3-month treatment with drospirenone $(3 \mathrm{mg})$ and ethinylestradiol $(30 \mu \mathrm{g})$ reduces ovarian vascularization both in control and PCOS patients, with a stronger effect on PCOS population compared to control patients 
(Okyay et al. 2014). The results presented in this study promote further research into the effect of OCPs on ovarian vascularization.

In our laboratory, we investigated the effect of local VEGF inhibition (Abramovich et al. 2012) and PDGFBB administration (Di Pietro et al. 2016) under the ovarian bursa of rats, previously injected with DHEA to mimic PCOS. We found that inhibiting ovarian VEGF by using a soluble receptor partially restores the accumulation of small follicles and reduces the formation of cysts in the PCOS rats. This strategy also increases ovulation and improves follicular development (Abramovich et al. 2012). Similarly, PDGFBB ovarian administration to PCOS rats partially restores primordial follicle accumulation, reduces cysts and increases corpora lutea formation. It also decreases ovarian VEGF levels and improves follicular vascularization (Di Pietro et al. 2016). Taken together, these findings support the hypothesis that normalization of ovarian angiogenesis can be considered a new strategy for improving follicular development, selection of the dominant follicle and ovulation in PCOS.

\section{Vasculogenesis dysfunctions in PCOS}

Endothelial progenitor cells (EPCs) are bone marrowderived mononuclear cells that possess the ability to migrate to neovascularization sites and once there proliferate and differentiate into mature endothelial cells, participating in the formation of the new blood vessels. EPC alterations in number and/or function have been involved in the development of atherosclerosis and impaired glucose metabolism (Fadini et al. 2010). Given the high risk of developing endothelial dysfunction combined with the vascular deregulation present in women with PCOS, EPCs have become candidates possibly involved in these pathogenic processes (DessaptBaradez et al. 2011, Kao et al. 2013). In this regard, Dessapt-Baradez et al. have investigated the number and function of EPCs in non-obese women with PCOS compared to age and BMI-matched control women. Decreased circulating number and impaired function of EPCs were found in the PCOS group compared to the control group. These alterations are closely related to the increased risk of CVD and vascular aging in patients with PCOS (Dessapt-Baradez et al. 2011).

Based on these results, improving EPC function in PCOS ovaries may provide important insights to enhance ovarian vessel formation in these patients. More studies should be undertaken to confirm this hypothesis.

\section{Conclusions and future remarks}

In the last years, continuous research has contributed to better characterize PCOS and set new diagnostic and therapeutic strategies for its management. Ovarian angiogenesis dysfunction has thrived as a new field in the study of PCOS, not only as a marker of CVD but also as a marker of ovarian deregulation. An increasing body of evidence demonstrates that the impairment of blood vessel development is a central feature in the PCOS pathophysiology, contributing to the most important traits of the syndrome, such as infertility. Therefore, strategies that target angiogenesis in women with PCOS are being evaluated in order to manage different aspects of this syndrome. Moreover, ovarian angiogenic imbalance has been proposed as a new tool in PCOS diagnosis. Improving ovarian angiogenesis in women with PCOS appears to be a promising strategy to enhance ovulation and follicular development in these patients. Further studies are required to clarify the role of angiogenesis in PCOS and to develop new potential therapies.

\section{Declaration of interest}

The authors declare that there is no conflict of interest that could be perceived as prejudicing the impartiality of this review.

\section{Funding}

This work was supported by the ANPCyT (PICT 2015-0114).

\section{References}

Abd El Aal DE, Mohamed SA, Amine AF \& Meki AR 2005 Vascular endothelial growth factor and insulin-like growth factor-1 in polycystic ovary syndrome and their relation to ovarian blood flow. European Journal of Obstetrics and Gynecology and Reproductive Biology 118 219-224. (https://doi.org/10.1016/j.ejogrb.2004.07.024)

Abdelsaid M, Kaczmarek J, Coucha M \& Ergul A 2014 Dual endothelin receptor antagonism with bosentan reverses established vascular remodeling and dysfunctional angiogenesis in diabetic rats: relevance to glycemic control. Life Sciences 118 268-273. (https://doi.org/10.1016/j. Ifs.2014.01.008)

Abramovich D, Rodriguez CA, Hernandez F, Tesone $M$ \& Parborell $F$ 2009 Spatiotemporal analysis of the protein expression of angiogenic factors and their related receptors during folliculogenesis in rats with and without hormonal treatment. Reproduction 137 309-320. (https:// doi.org/10.1530/REP-08-0130)

Abramovich D, Irusta G, Bas D, Cataldi NI, Parborell F \& Tesone M 2012 Angiopoietins/TIE2 system and VEGF are involved in ovarian function in a DHEA rat model of polycystic ovary syndrome. Endocrinology $\mathbf{1 5 3}$ 3446-3456. (https://doi.org/10.1210/en.2012-1105)

Agrawal R, Conway G, Sladkevicius P, Tan SL, Engmann L, Payne N, Bekir J, Campbell S \& Jacobs H 1998a Serum vascular endothelial growth factor and Doppler blood flow velocities in in vitro fertilization: relevance to ovarian hyperstimulation syndrome and polycystic ovaries. Fertility and Sterility 70 651-658. (https://doi.org/10.1016/S0015-0282(98)00249-0)

Agrawal R, Sladkevicius P, Engmann L, Conway GS, Payne NN, Bekis J, Tan SL, Campbell S \& Jacobs HS 1998b Serum vascular endothelial growth factor concentrations and ovarian stromal blood flow are increased in women with polycystic ovaries. Human Reproduction $\mathbf{1 3}$ 651-655. (https://doi.org/10.1093/humrep/13.3.651)

Albiges L, Salem M, Rini B \& Escudier B 2011 Vascular endothelial growth factor-targeted therapies in advanced renal cell carcinoma. Hematology/Oncology Clinics of North America 25 813-833. (https:// doi.org/10.1016/j.hoc.2011.04.006)

Alcazar JL \& Kudla MJ 2012 Ovarian stromal vessels assessed by spatiotemporal image correlation-high definition flow in women with polycystic ovary syndrome: a case-control study. Ultrasound in Obstetrics and Gynecology 40 470-475. (https://doi.org/10.1002/uog.11187) 
Almawi WY, Gammoh E, Malalla ZH \& Al Madhi SA 2016 Analysis of VEGFA variants and changes in VEGF levels underscores the contribution of VEGF to polycystic ovary syndrome. PLoS ONE 11 e0165636. (https:// doi.org/10.1371/journal.pone.0165636)

Amin AF, Abd el-Aal DE, Darwish AM \& Meki AR 2003 Evaluation of the impact of laparoscopic ovarian drilling on Doppler indices of ovarian stromal blood flow, serum vascular endothelial growth factor, and insulin-like growth factor-1 in women with polycystic ovary syndrome. Fertility and Sterility 79 938-941. (https://doi.org/10.1016/S00150282(02)04849-5)

Artini PG, Monti M, Matteucci C, Valentino V, Cristello F \& Genazzani AR 2006 Vascular endothelial growth factor and basic fibroblast growth factor in polycystic ovary syndrome during controlled ovarian hyperstimulation. Gynecological Endocrinology 22 465-470. (https:// doi.org/10.1080/09513590600906607)

Artini PG, Ruggiero M, Parisen Toldin MR, Monteleone P, Monti M, Cela V \& Genazzani AR 2009 Vascular endothelial growth factor and its soluble receptor in patients with polycystic ovary syndrome undergoing IVF. Human Fertility 12 40-44. (https://doi. org/10.1080/14647270802621358)

Battaglia C, Artini PG, D'Ambrogio G, Genazzani AD \& Genazzani AR 1995 The role of color Doppler imaging in the diagnosis of polycystic ovary syndrome. American Journal of Obstetrics and Gynecology 172 108-113. (https://doi.org/10.1016/0002-9378(95)90094-2)

Battaglia DE, Goodwin P, Klein NA \& Soules MR 1996 Influence of maternal age on meiotic spindle assembly in oocytes from naturally cycling women. Human Reproduction 11 2217-2222. (https://doi. org/10.1093/oxfordjournals.humrep.a019080)

Battaglia C, Artini PG, Genazzani AD, Gremigni R, Salvatori M, Sgherzi MR, Giulini S, Lombardo M \& Volpe A 1997 Color Doppler analysis in oligo- and amenorrheic women with polycystic ovary syndrome. Gynecological Endocrinology 11 105-110. (https://doi. org/10.3109/09513599709152520)

Bauditz J \& Lochs H 2007 Angiogenesis and vascular malformations: antiangiogenic drugs for treatment of gastrointestinal bleeding. World Journal of Gastroenterology 13 5979-5984. (https://doi.org/10.3748/ wjg.v13.45.5979)

Betsholtz C 2004 Insight into the physiological functions of PDGF through genetic studies in mice. Cytokine and Growth Factor Reviews 15 215-228. (https://doi.org/10.1016/j.cytogfr.2004.03.005)

Cao Y, Chen H, Zhou L, Chiang MK, Anand-Apte B, Weatherbee JA, Wang Y, Fang F, Flanagan JG \& Tsang ML 1996 Heterodimers of placenta growth factor/vascular endothelial growth factor. Endothelial activity, tumor cell expression, and high affinity binding to Flk-1/KDR. Journal of Biological Chemistry 271 3154-3162. (https://doi.org/10.1074/ jbc.271.6.3154)

Carmina E, Orio F, Palomba S, Longo RA, Lombardi G \& Lobo RA 2005 Ovarian size and blood flow in women with polycystic ovary syndrome and their correlations with endocrine parameters. Fertility and Sterility 84 413-419. (https://doi.org/10.1016/j.fertnstert.2004.12.061)

Dallaglio K, Bruno A, Cantelmo AR, Esposito AI, Ruggiero L, Orecchioni S, Calleri A, Bertolini F, Pfeffer U, Noonan DM et al. 2014 Paradoxic effects of metformin on endothelial cells and angiogenesis. Carcinogenesis 35 1055-1066. (https://doi.org/10.1093/carcin/bgu001)

Davis S, Aldrich TH, Jones PF, Acheson A, Compton DL, Jain V, Ryan TE, Bruno J, Radziejewski C, Maisonpierre PC et al. 1996 Isolation of angiopoietin-1, a ligand for the TIE2 receptor, by secretion-trap expression cloning. Cell 87 1161-1169. (https://doi.org/10.1016/S00928674(00)81812-7)

Dessapt-Baradez C, Reza M, Sivakumar G, Hernandez-Fuentes M, Markakis K, Gnudi L \& Karalliedde J 2011 Circulating vascular progenitor cells and central arterial stiffness in polycystic ovary syndrome. PLoS ONE 6 e20317. (https://doi.org/10.1371/journal.pone.0020317)

Di Pietro M, Parborell F, Irusta G, Pascuali N, Bas D, Bianchi MS, Tesone M \& Abramovich D 2015 Metformin regulates ovarian angiogenesis and follicular development in a female polycystic ovary syndrome rat model. Endocrinology 156 1453-1463. (https://doi.org/10.1210/en.2014-1765)

Di Pietro M, Scotti L, Irusta G, Tesone M, Parborell F \& Abramovich D 2016 Local administration of platelet-derived growth factor B (PDGFB) improves follicular development and ovarian angiogenesis in a rat model of polycystic ovary syndrome. Molecular and Cellular Endocrinology 433 47-55. (https://doi.org/10.1016/j.mce.2016.05.022)
El Behery MM, Diab AE, Mowafy H, Ebrahiem MA \& Shehata AE 2011 Effect of laparoscopic ovarian drilling on vascular endothelial growth factor and ovarian stromal blood flow using 3-dimensional power Doppler. International Journal of Gynecology and Obstetrics 112 119-121. (https://doi.org/10.1016/j.ijgo.2010.08.018)

Elmashad Al 2011 Impact of laparoscopic ovarian drilling on anti-Mullerian hormone levels and ovarian stromal blood flow using three-dimensional power Doppler in women with anovulatory polycystic ovary syndrome. Fertility and Sterility 95 2342-2346. (https:// doi.org/10.3892/or.2012.1745)

Fadini GP, Boscaro E, de Kreutzenberg S, Agostini C, Seeger F, Dimmeler S, Zeiher A, Tiengo A \& Avogaro A 2010 Time course and mechanisms of circulating progenitor cell reduction in the natural history of type 2 diabetes. Diabetes Care 33 1097-1102. (https://doi.org/10.2337/dc09-1999)

Ferrara N, Frantz G, LeCouter J, Dillard-Telm L, Pham T, Draksharapu A, Giordano T \& Peale F 2003 Differential expression of the angiogenic factor genes vascular endothelial growth factor (VEGF) and endocrine gland-derived VEGF in normal and polycystic human ovaries. American Journal of Pathology 162 1881-1893. (https://doi.org/10.1016/S00029440(10)64322-2)

Gale NW, Thurston G, Hackett SF, Renard R, Wang Q, McClain J, Martin C, Witte C, Witte MH, Jackson D, et al. 2002 Angiopoietin-2 is required for postnatal angiogenesis and lymphatic patterning, and only the latter role is rescued by Angiopoietin-1. Developmental Cell 3 411-423. (https:// doi.org/10.1016/S1534-5807(02)00217-4)

Giampaolino P, Morra I, De Rosa N, Cagnacci A, Pellicano M, Di Carlo C, Nappi C \& Bifulco G 2017 Impact of transvaginal hydrolaparoscopy ovarian drilling on ovarian stromal blood flow and ovarian volume in clomiphene citrate-resistant PCOS patients: a case-control study. Gynecological Endocrinology 33 690-693. (https://doi.org/10.1080/09 513590.2017.1310837)

Gomez R, Soares SR, Busso C, Garcia-Velasco JA, Simon C \& Pellicer A 2010 Physiology and pathology of ovarian hyperstimulation syndrome. Seminars in Reproductive Medicine 28 448-457. (https://doi. org/10.1055/s-0030-1265670)

Gospodarowicz D, Ferrara N, Schweigerer L \& Neufeld G 1987 Structural characterization and biological functions of fibroblast growth factor. Endocrine Reviews 8 95-114. (https://doi.org/10.1210/edrv-8-2-95)

Greenblatt E \& Casper RF 1987 Endocrine changes after laparoscopic ovarian cautery in polycystic ovarian syndrome. American Journal of Obstetrics and Gynecology 156 279-285. (https://doi.org/10.1016/00029378(87)90268-7)

Guruvaiah P, Govatati S, Reddy TV, Lomada D, Deenadayal M, Shivaji S \& Bhanoori M 2014 The VEGF +405 G>C 5' untranslated region polymorphism and risk of PCOS: a study in the South Indian Women. Journal of Assisted Reproduction and Genetics 31 1383-1389. (https:// doi.org/10.1007/s10815-014-0310-4)

Hazzard TM \& Stouffer RL 2000 Angiogenesis in ovarian follicular and luteal development. Bailliere's Best Practice and Research: Clinical Obstetrics and Gynaecology 6 883-900. (https://doi.org/10.1053/ beog.2000.0133)

Heldin CH \& Westermark B 1999 Mechanism of action and in vivo role of platelet-derived growth factor. Physiological Reviews 79 1283-1316. (https://doi.org/10.1152/physrev.1999.79.4.1283)

Hoch RV \& Soriano P 2003 Roles of PDGF in animal development. Development 130 4769-4784. (https://doi.org/10.1242/dev.00721)

Hueb CK, Dias Junior JA, Abrao MS \& Filho EK 2015 Drilling: medical indications and surgical technique. Revista Da Associacao Medica Brasileira 61 530-535. (https://doi.org/10.1590/1806-9282.61.06.530)

Irusta G, Abramovich D, Parborell F \& Tesone M 2010 Direct survival role of vascular endothelial growth factor (VEGF) on rat ovarian follicular cells. Molecular and Cellular Endocrinology 325 93-100. (https://doi. org/10.1016/j.mce.2010.04.018)

Johnson N 2011 Metformin is a reasonable first-line treatment option for non-obese women with infertility related to anovulatory polycystic ovary syndrome - a meta-analysis of randomised trials. Australian and New Zealand Journal of Obstetrics and Gynaecology 51 125-129. (https://doi. org/10.1111/j.1479-828X.2010.01274.x)

Jungheim ES \& Odibo AO 2010 Fertility treatment in women with polycystic ovary syndrome: a decision analysis of different oral ovulation induction agents. Fertility and Sterility 94 2659-2664. (https://doi.org/10.1016/j. fertnstert.2010.03.077) 
Kamat BR, Brown LF, Manseau EJ, Senger DR \& Dvorak HF 1995 Expression of vascular permeability factor/vascular endothelial growth factor by human granulosa and theca lutein cells. Role in corpus luteum development. American Journal of Pathology 146 157-165.

Kao YH, Chiu WC, Hsu MI \& Chen YJ 2013 Endothelial progenitor cell dysfunction in polycystic ovary syndrome: implications for the genesis of cardiovascular diseases. International Journal of Fertility and Sterility 6 208-213.

Karimzadeh L, Nabiuni M, Kouchesfehani HM, Adham H, Bagheri A \& Sheikholeslami A 2013 Effect of bee venom on IL-6, COX-2 and VEGF levels in polycystic ovarian syndrome induced in Wistar rats by estradiol valerate. Journal of Venomous Animals and Toxins including Tropical Diseases 19 32. (https://doi.org/10.1186/16789199-19-32)

Kendall RL, Wang G \& Thomas KA 1996 Identification of a natural soluble form of the vascular endothelial growth factor receptor, FLT1 , and its heterodimerization with KDR. Biochemical and Biophysical Research Communications 226 324-328. (https://doi.org/10.1006/ bbrc.1996.1355)

Khattab S, Fotouh IA, Mohesn IA, Metwally M \& Moaz M 2006 Use of metformin for prevention of ovarian hyperstimulation syndrome: a novel approach. Reproductive BioMedicine Online 13 194-197. (https://doi. org/10.1016/S1472-6483(10)60614-1)

Kjotrod SB, Carlsen SM, Rasmussen PE, Holst-Larsen T, Mellembakken J, Thurin-Kjellberg A, Haapaniemikouru K, Morin-Papunen L, Humaidan P, Sunde A et al. 2011 Use of metformin before and during assisted reproductive technology in non-obese young infertile women with polycystic ovary syndrome: a prospective, randomized, doubleblind, multi-centre study. Human Reproduction 26 2045-2053. (https:// doi.org/10.1093/humrep/der154)

Laiho M, DeCaprio JA, Ludlow JW, Livingston DM \& Massague J 1990 Growth inhibition by TGF-beta linked to suppression of retinoblastoma protein phosphorylation. Cell 62 175-185. (https://doi. org/10.1016/0092-8674(90)90251-9)

Lee EJ, Oh B, Lee JY, Kimm K, Park JM \& Baek KH 2008 Association study between single nucleotide polymorphisms in the VEGF gene and polycystic ovary syndrome. Fertility and Sterility 89 1751-1759. (https:// doi.org/10.1016/j.fertnstert.2007.06.049)

Leite DO, Hamm A \& Mazzone M 2011 Growing tumor vessels: more than one way to skin a cat - implications for angiogenesis targeted cancer therapies. Molecular Aspects of Medicine 32 71-87. (https://doi. org/10.1016/j.mam.2011.04.001)

Liao H, Zhou Q, Gu Y, Duan T \& Feng Y 2012 Luteinizing hormone facilitates angiogenesis in ovarian epithelial tumor cells and metformin inhibits the effect through the mTOR signaling pathway. Oncology Reports 27 1873-1878.

Liu M, Gao J, Zhang Y, Li P, Wang H, Ren X \& Li C 2015 Serum levels of TSP-1, NF-kappaB and TGF-beta1 in polycystic ovarian syndrome (PCOS) patients in northern China suggest PCOS is associated with chronic inflammation. Clinical Endocrinology 83 913-922. (https://doi. org/10.1111/cen.12951)

Maisonpierre PC, Suri C, Jones PF, Bartunkova S, Wiegand SJ, Radziejewski C, Compton D, McClain J, Aldrich TH \& Papadopoulos N 1997 Angiopoietin-2, a natural antagonist for Tie2 that disrupts in vivo angiogenesis. Science 277 55-60. (https://doi.org/10.1126/ science.277.5322.55)

Makled AK, El Sherbiny M \& Elkabarity R 2014 Assessment of ovarian stromal blood flow after metformin treatment in women with polycystic ovary syndrome. Archives of Gynecology and Obstetrics 289 883-891. (https://doi.org/10.1007/s00404-013-3057-8)

Mitchell P 2011 A systematic review of the efficacy and safety outcomes of anti-VEGF agents used for treating neovascular age-related macular degeneration: comparison of ranibizumab and bevacizumab. Current Medical Research and Opinion 27 1465-1475. (https://doi.org/10.118 5/03007995.2011.585394)

Moll E, van der Veen F \& van Wely M 2007 The role of metformin in polycystic ovary syndrome: a systematic review. Human Reproduction Update 13 527-537. (https://doi.org/10.1093/humupd/dmm026)

Nader S \& Diamanti-Kandarakis E 2007 Polycystic ovary syndrome, oral contraceptives and metabolic issues: new perspectives and a unifying hypothesis. Human Reproduction 22 317-322. (https://doi.org/10.1093/ humrep/del407)
Nilsson EE, Detzel C \& Skinner MK 2006 Platelet-derived growth factor modulates the primordial to primary follicle transition. Reproduction 131 1007-1015. (https://doi.org/10.1530/rep.1.00978)

Nissen LJ, Cao R, Hedlund EM, Wang Z, Zhao X, Wetterskog D, Funa K, Brakenhielm E \& Cao Y 2007 Angiogenic factors FGF2 and PDGFBB synergistically promote murine tumor neovascularization and metastasis. Journal of Clinical Investigation 117 2766-2777. (https://doi. org/10.1172/JCI32479)

Norman RJ, Dewailly D, Legro RS \& Hickey TE 2007 Polycystic ovary syndrome. Lancet $\mathbf{3 7 0}$ 685-697. (https://doi.org/10.1016/S01406736(07)61345-2)

Okyay E, Gode F, Acet F, Bodur T, Cagliyan E, Sahan C, Posaci C \& Gulekli B 2014 The effect of drospirenone (3 mg) with ethinyl estradiol (30 mcg) containing pills on ovarian blood flows in women with polycystic ovary syndrome: a case controlled study. European Journal of Obstetrics and Gynecology and Reproductive Biology 180 93-99. (https://doi. org/10.1016/j.ejogrb.2014.06.027)

Ollauri-Ibanez C, Lopez-Novoa JM \& Pericacho M 2017 Endoglinbased biological therapy in the treatment of angiogenesis-dependent pathologies. Expert Opinion on Biological Therapy 17 1053-1063. (https://doi.org/10.1080/14712598.2017.1346607)

Palomba S, Falbo A, Carrillo L, Villani MT, Orio F, Russo T, Di Cello A, Cappiello F, Capasso S, Tolino A et al. 2011 Metformin reduces risk of ovarian hyperstimulation syndrome in patients with polycystic ovary syndrome during gonadotropin-stimulated in vitro fertilization cycles: a randomized, controlled trial. Fertility and Sterility 96 1384-1390. (https://doi.org/10.1016/j.fertnstert.2011.09.020)

Palomba S, Falbo A \& La Sala GB 2013 Effects of metformin in women with polycystic ovary syndrome treated with gonadotrophins for in vitro fertilisation and intracytoplasmic sperm injection cycles: a systematic review and meta-analysis of randomised controlled trials. BJOG 120 267-276. (https://doi.org/10.1111/1471-0528.12070)

Palomba S, Falbo A \& La Sala GB 2014 Metformin and gonadotropins for ovulation induction in patients with polycystic ovary syndrome: a systematic review with meta-analysis of randomized controlled trials. Reproductive Biology and Endocrinology 12 3. (https://doi. org/10.1186/1477-7827-12-3)

Pandya NM, Dhalla NS \& Santani DD 2006 Angiogenesis - a new target for future therapy. Vascular Pharmacology 44 265-274. (https://doi. org/10.1016/j.vph.2006.01.005)

Park JE, Chen HH, Winer J, Houck KA \& Ferrara N 1994 Placenta growth factor. Potentiation of vascular endothelial growth factor bioactivity, in vitro and in vivo, and high affinity binding to Flt-1 but not to Flk-1/KDR. Journal of Biological Chemistry 269 25646-25654.

Pascuali N, Scotti L, Abramovich D, Irusta G, Di Pietro M, Bas D, Tesone M \& Parborell F 2015 Inhibition of platelet-derived growth factor (PDGF) receptor affects follicular development and ovarian proliferation, apoptosis and angiogenesis in prepubertal eCG-treated rats. Molecular and Cellular Endocrinology 412 148-158. (https://doi.org/10.1016/j. mce.2015.04.021)

Pinkas H, Fisch B, Rozansky G, Felz C, Kessler-Icekson G, Krissi H, Nitke S, Ao A \& Abir R 2008 Platelet-derived growth factors (PDGF-A and -B) and their receptors in human fetal and adult ovaries. Molecular Human Reproduction 14 199-206. (https://doi.org/10.1093/molehr/gan011)

Raja-Khan N, Kunselman AR, Demers LM, Ewens KG, Spielman RS \& Legro RS 2010 A variant in the fibrillin-3 gene is associated with TGFbeta and inhibin B levels in women with polycystic ovary syndrome. Fertility and Sterility 94 2916-2919. (https://doi.org/10.1016/j. fertnstert.2010.05.047)

Rattan R, Graham RP, Maguire JL, Giri S \& Shridhar V 2011 Metformin suppresses ovarian cancer growth and metastasis with enhancement of cisplatin cytotoxicity in vivo. Neoplasia 13 483-491. (https://doi. org/10.1593/neo.11148)

Resende AV, Mendes MC, Dias DM, Mendonca HC, Gomes Premoli AC, Reis RM \& Berezowski AT 2001 Doppler study of the uterine arteries and ovarian stroma in patients with polycystic ovary syndrome. Gynecologic and Obstetric Investigation 52 153-157. (https://doi. org/10.1159/000052964)

Reynolds LP \& Redmer DA 1998 Expression of the angiogenic factors, basic fibroblast growth factor and vascular endothelial growth factor, in the ovary. Journal of Animal Science 76 1671-1681. (https://doi. org/10.2527/1998.7661671x) 
Ribeiro JA, Messias A \& Jorge R 2011 Antiangiogenic drugs and advanced proliferative diabetic retinopathy. Arquivos Brasileiros de Oftalmologia 74 143-146. (https://doi.org/10.1590/S0004-27492011000200017)

Rosenfield RL \& Ehrmann DA 2016 The pathogenesis of polycystic ovary syndrome (PCOS): the hypothesis of PCOS as functional ovarian hyperandrogenism revisited. Endocrine Reviews 37 467-520. (https:// doi.org/10.1210/er.2015-1104)

Rotterdam ESHRE/ASRM-Sponsored PCOS Consensus Workshop Group 2004 Revised 2003 consensus on diagnostic criteria and long-term health risks related to polycystic ovary syndrome (PCOS). Human Reproduction 19 41-47.

Satterwhite DJ \& Moses HL 1994 Mechanisms of transforming growth factor-beta 1-induced cell cycle arrest. Invasion Metastasis 14 309-318.

Scotti L, Parborell F, Irusta G, De Zuñiga I, Bisioli C, Pettorossi H, Tesone M \& Abramovich D 2014 Platelet-derived growth factor BB and DD and angiopoietin1 are altered in follicular fluid from polycystic ovary syndrome patients. Molecular Reproduction and Development 81 748-756. (https://doi.org/10.1002/mrd.22343)

Shank JJ, Yang K, Ghannam J, Cabrera L, Johnston CJ, Reynolds RK \& Buckanovich RJ 2012 Metformin targets ovarian cancer stem cells in vitro and in vivo. Gynecologic Oncology 127 390-397. (https://doi. org/10.1016/j.ygyno.2012.07.115)

Sharma PS, Sharma R \& Tyagi T 2011 VEGF/VEGFR pathway inhibitors as anti-angiogenic agents: present and future. Current Cancer Drug Targets 11 624-653. (https://doi.org/10.2174/156800911795655985)

Shim WS, Ho IA \& Wong PE 2007 Angiopoietin: a TIE(d) balance in tumor angiogenesis. Molecular Cancer Research 5 655-65. (https://doi. org/10.1158/1541-7786.MCR-07-0072)

Sleer LS \& Taylor CC 2007 Cell-type localization of platelet-derived growth factors and receptors in the postnatal rat ovary and follicle. Biology of Reproduction 76 379-390. (https://doi.org/10.1095/ biolreprod.105.046854)

Socinski MA 2011 Multitargeted receptor tyrosine kinase inhibition: an antiangiogenic strategy in non-small cell lung cancer. Cancer Treatment Reviews 37 611-617. (https://doi.org/10.1016/j.ctrv.2011.04.003)

Sova H, Morin-Papunen L, Puistola U \& Karihtala P 2010 Distinctively low levels of serum 8-hydroxydeoxyguanosine in women with polycystic ovary syndrome. Fertility and Sterility 94 2670-2673. (https://doi. org/10.1016/j.fertnstert.2010.03.049)

Stein IF \& Leventhal ML 1935 Amenorrhea associated with bilateral polycystic ovaries. American Journal of Obstetrics and Gynecology 129 181-191. (https://doi.org/10.1016/S0002-9378(15)30642-6)

Tal R, Seifer DB, Grazi RV \& Malter HE 2013a Angiopoietin-1 and angiopoietin-2 are altered in polycystic ovarian syndrome (PCOS) during controlled ovarian stimulation. Vascular Cell 5 18. (https://doi. org/10.1186/2045-824X-5-18)

Tal R, Seifer DB, Shohat-Tal A, Grazi RV \& Malter HE 2013b Transforming growth factor-beta1 and its receptor soluble endoglin are altered in polycystic ovary syndrome during controlled ovarian stimulation. Fertility and Sterility $\mathbf{1 0 0}$ 538-543. (https://doi.org/10.1016/j. fertnstert.2013.04.022)

Tal R, Seifer DB, Grazi RV \& Malter HE 2014 Follicular fluid placental growth factor is increased in polycystic ovarian syndrome: correlation with ovarian stimulation. Reproductive Biology and Endocrinology 12 82. (https://doi.org/10.1186/1477-7827-12-82)

Tan BK, Adya R, Chen J, Farhatullah S, Heutling D, Mitchell D, Lehnert H \& Randeva HS 2009 Metformin decreases angiogenesis via
NF-kappaB and Erk1/2/Erk5 pathways by increasing the antiangiogenic thrombospondin-1. Cardiovascular Research 83 566-574. (https://doi. org/10.1093/cvr/cvp131)

Tan BK, Adya R, Farhatullah S, Chen J, Lehnert H \& Randeva HS 2010 Metformin treatment may increase omentin-1 levels in women with polycystic ovary syndrome. Diabetes 59 3023-3031. (https://doi. org/10.2337/db10-0124)

Tang T, Lord JM, Norman RJ, Yasmin E \& Balen AH 2012 Insulin-sensitising drugs (metformin, rosiglitazone, pioglitazone, D-chiro-inositol) for women with polycystic ovary syndrome, oligo amenorrhoea and subfertility. Cochrane Database of Systematic Reviews 5 CD003053. (https://doi.org/10.1002/14651858.CD003053.pub5)

Thessaloniki ESHRE/ASRM-Sponsored PCOS Consensus Workshop Group 2008 Consensus on infertility treatment related to polycystic ovary syndrome. Human Reproduction 23 462-477. (https://doi.org/10.1093/ humrep/dem426)

Turner N \& Grose R 2010 Fibroblast growth factor signalling: from development to cancer Nature Reviews Cancer 2 116-129. (https://doi. org/10.1038/nrc2780)

Vrbikova J \& Cibula D 2005 Combined oral contraceptives in the treatment of polycystic ovary syndrome. Human Reproduction Update 11 277-291. (https://doi.org/10.1093/humupd/dmi005)

Vural P, Kusku-Kiraz Z, Dogru-Abbasoglu S, Cil E, Karadag B, Akgul C \& Uysal M 2009 Vascular endothelial growth factor -2578 A/C, -460 T/C and $+405 \mathrm{G} / \mathrm{C}$ polymorphisms in polycystic ovary syndrome. European Journal of Obstetrics and Gynecology and Reproductive Biology. (https:// doi.org/10.1016/j.ejogrb.2009.06.026)

Waltenberger J, Claesson-Welsh L, Siegbahn A, Shibuya M \& Heldin CH 1994 Different signal transduction properties of KDR and Flt1, two receptors for vascular endothelial growth factor. Journal of Biological Chemistry $26926988-26995$.

Wang L, Qi H, Baker PN, Zhen Q, Zeng Q, Shi R, Tong C \& Ge Q 2017 Altered circulating inflammatory cytokines are associated with anovulatory polycystic ovary syndrome (PCOS) women resistant to clomiphene citrate treatment. Medical Science Monitor 23 1083-1089. (https://doi.org/10.12659/MSM.901194)

Wu B, Li S, Sheng L, Zhu J, Gu L, Shen H, La D, Hambly BD, Bao S \& Di W 2012 Metformin inhibits the development and metastasis of ovarian cancer. Oncology Reports 28 903-908. (https://doi.org/10.3892/ or.2012.1890)

Yang EY \& Moses HL 1990 Transforming growth factor beta 1-induced changes in cell migration, proliferation, and angiogenesis in the chicken chorioallantoic membrane. Journal of Cell Biology 111 731-741. (https:// doi.org/10.1083/jcb.111.2.731)

Zaidi J, Campbell S, Pittrof R, Kyei-Mensah A, Shaker A, Jacobs HS \& Tan SL 1995 Ovarian stromal blood flow in women with polycystic ovaries - a possible new marker for diagnosis? Human Reproduction 10 1992-1996. (https://doi.org/10.1093/oxfordjournals.humrep.a136222)

Received 2 October 2017

First decision 9 November 2017

Revised manuscript received 29 December 2017

Accepted 30 January 2018 